\title{
Erratum to: JAK-STAT Signaling as a Target for Inflammatory and Autoimmune Diseases: Current and Future Prospects
}

\author{
Shubhasree Banerjee ${ }^{1} \cdot$ Ann Biehl $^{2} \cdot$ Massimo Gadina $^{3}$. \\ Sarfaraz Hasni ${ }^{4} \cdot$ Daniella M. Schwartz ${ }^{5}$
}

Published online: 12 June 2017

(c) Springer International Publishing Switzerland 2017

\section{Erratum to: Drugs (2017) 77:521-546 \\ DOI 10.1007/s40265-017-0701-9}

Page 539, Table 6 Janus Kinase Inhibitors in preclinical and early clinical development

The first row/entry which currently reads:

\begin{tabular}{llll}
\hline Drug & Specificity & Clinical Status & Diseases \\
\hline OP0155 [199] & JAK3 & Preclinical & Rat adjuvant induced arthritis \\
\hline
\end{tabular}

Should read:

\begin{tabular}{llll}
\hline Drug & Specificity & Clinical Status & Diseases \\
\hline PF-06651600 [199] & JAK3 & $\begin{array}{c}\text { Phase 2 (ClinicalTrials.gov. NCT02969044, } \\
\text { NCT02974868, NCT02958865) }\end{array}$ & $\begin{array}{c}\text { Rheumatoid arthritis, alopecia areata, } \\
\text { ulcerative colitis }\end{array}$ \\
\hline
\end{tabular}

The online version of the original article can be found under doi:10.1007/s40265-017-0701-9.

\section{Shubhasree Banerjee}

shubhasree.banerjee@nih.gov

1 Rheumatology Fellowship and Training Branch, National Institute of Arthritis Musculoskeletal and Skin Diseases,

National Institutes of Health, Bethesda, USA

2 Clinical Center, National Institutes of Health, Bethesda, USA

3 Translational Immunology Section, National Institute of Arthritis Musculoskeletal and Skin diseases, National Institutes of Health, Bethesda, USA
4 Lupus Clinical Research Program, National Institute of Arthritis Musculoskeletal and Skin diseases, National Institutes of Health, Bethesda, USA

5 Molecular Immunology and Inflammation Branch, National Institute of Arthritis Musculoskeletal and Skin diseases, National Institutes of Health, Bethesda, USA 